\title{
BMJ Open Thromboprophylaxis only during hospitalisation in fast-track hip and knee arthroplasty, a prospective cohort study
}

Christoffer C Jørgensen, ${ }^{1}$ Michael K Jacobsen, ${ }^{2}$ Kjeld Soeballe, ${ }^{2}$

Torben B Hansen, ${ }^{3}$ Henrik Husted, ${ }^{4}$ Per Kjærsgaard-Andersen, ${ }^{5}$ Lars T Hansen, ${ }^{6}$ Mogens B Laursen, ${ }^{7}$ Henrik Kehlet ${ }^{1}$

To cite: Jørgensen CC, Jacobsen MK, Soeballe K, et al. Thromboprophylaxis only during hospitalisation in fast-track hip and knee arthroplasty, a prospective cohort study. BMJ Open 2013;3:e003965. doi:10.1136/ bmjopen-2013-003965

- Prepublication history and additional material for this paper is available online. To view these files please visit the journal online (http://dx.doi.org/10.1136/ bmjopen-2013-003965)

All authors are members of The Lundbeck Foundation Centre for Fast-track Hip and Knee Replacement 2100 Copenhagen, Denmark.

Received 6 September 2013 Revised 28 October 2013 Accepted 1 November 2013

CrossMark

For numbered affiliations see end of article.

Correspondence to

Dr Christoffer C Jørgensen; christoffer.calov.joergensen@ regionh.dk

\section{ABSTRACT}

Objectives: International guidelines recommend thrombosis prophylaxis after total hip arthroplasty (THA) and total knee arthroplasty (TKA) for up to 35 days. However, previous studies often have hospital stays (length of stay; LOS) of 8-12 days and not considering early mobilisation, which may reduce incidence of venous thromboembolic events (VTE). We investigated the incidence of any symptomatic thromboembolic events (TEEs) with only in-hospital prophylaxis if LOS $\leq 5$ days after fast-track THA and TKA.

Design: A prospective descriptive multicentre cohort study in fast-track THA and TKA from February 2010 to December 2011, with complete 90-day follow-up through the Danish National Patient Registry and patient files.

Setting: 6 Danish high-volume centres with a similar standardised fast-track setup, including spinal anaesthesia, opioid-sparing analgesia, early mobilisation, functional discharge criteria and discharge to own home.

Participants: 4924 consecutive unselected unilateral primary THA and TKAs in patients $\geq 18$ years with no preoperative use of continuous 'potent' anticoagulative therapy (vitamin $\mathrm{K}$ antagonists).

Exposure: Prophylaxis with low-molecular-weight heparin or factor Xa-inhibitors only during hospitalisation when $L O S \leq 5$ days.

Outcomes: Incidence of symptomatic TEE-related, VTErelated and VTE-related mortality $\leq 90$ days postoperatively.

Results: LOS $\leq 5$ days and thromboprophylaxis only during hospitalisation occurred in 4659 procedures (94.6\% of total). Median LOS and prophylaxis duration was 2 days (IQR $2-3)$ with $0.84 \%(95 \% \mathrm{Cl} 0.62 \%$ to $1.15 \%)$ TEE and $0.41 \%(0.26 \%$ to $0.64 \%)$ VTE during 90-day follow-up. VTE consisted of five pulmonary embolisms $(0.11 \%(0.05 \%$ to $0.25 \%))$ and 14 deep venous thrombosis $(0.30 \%(0.18 \%$ to $0.50 \%))$. There were four $(0.09 \%(0.04 \%$ to $0.23 \%))$ surgery-related deaths, of which $1(0.02 \%(0.00 \%$ to $0.12 \%))$ was due to pulmonary embolism, and $6(0.13 \%(0.06 \%$ to $0.28 \%))$ deaths of unknown causes after discharge.

Conclusions: The low incidence of TEE and VTE suggests that in-hospital prophylaxis only, is safe in fasttrack THA and TKA patients with LOS of $\leq 5$ days. Guidelines on thromboprophylaxis may need reconsideration in fast-track elective surgery. Trial Registration: ClinicalTrials.gov: NCT01557725

\section{Strengths and limitations of this study}

- A prospective multicentre trial in a large cohort of consecutive unselected patients, with a standardised perioperative fast-track setup.

- Complete 90-day follow-up through the Danish National Patient Registry and patient files.

- Registration of thromboembolic event (TEE) was based on review of patient files, any TEE not mentioned in these would not have been registered.

\section{INTRODUCTION}

Venous thromboembolic events (VTE) such as deep venous thrombosis (DVT) and pulmonary embolism (PE) are well-documented risks in hospitalised patients. ${ }^{1}$ Surgery presents an independent risk factor for such events, due to both the surgical trauma and postoperative immobilisation. Consequently, guidelines for postoperative thromboprophylaxis have been developed in both general and orthopaedic surgery. ${ }^{2-4}$ However, the type and duration of prophylaxis following elective surgery is debatable. ${ }^{5-7}$ For example, the American College of Chest Physicians (ACCP) recommends either mechanical prophylaxis using intermittent pneumatic compressive devices (IPCD; grade 1C), or pharmacological prophylaxis (grade 1B), for up to 35 days (grade 2B) after total hip (THA) and knee arthroplasty (TKA), ${ }^{2}$ whereas the American Academy of Orthopedic Surgeons find the evidence inconclusive and decide the duration of thromboprophylaxis on an individual basis. 8 Much of the evidence regarding the duration of thromboprophylaxis after orthopaedic surgery has originated from large randomised clinical trials (RCTs) in THA and TKA with prophylaxis of 10-35 days, ${ }^{9-13}$ and these studies also contribute to guidelines in general surgery. ${ }^{3}$ However, the pathophysiological mechanisms of thrombosis have not been addressed in RCTs, which often have long length of stay (LOS) and lack focus on early 
mobilisation, despite the fact that early mobilisation per se may reduce the need for thromboprophylaxis. ${ }^{14}$

Fast-track surgery has been developed to improve recovery by using evidence-based care principles with multimodal opioid-sparing analgesia, reduction of the surgical stress-response, optimised fluid treatment, adjustment of the use of drains and catheters and early mobilisation. These efforts have resulted in improved outcome following various procedures such as colonic surgery and gynaecological procedures ${ }^{15}$ and major joint arthroplasty. ${ }^{16}$ It has been suggested that reassessment of thromboembolic risk in elective surgery is needed due to a few incidences of $\mathrm{VTE}^{5}{ }^{17}$; preliminary data have supported that fast-track THA and TKA may decrease the risk of VTE and thereby the need for prolonged prophylaxis. ${ }^{6}$ Consequently, we designed a large prospective cohort study in unselected consecutive patients having fast-track THA or TKA, with thromboprophylaxis only during hospitalisation when the LOS was $\leq 5$ days. We hypothesised that there would be no increase in symptomatic thromboembolic events (TEE) and VTE with prophylaxis only during hospitalisation compared with previous data with prophylaxis of 10-35 days.

\section{METHODS}

We investigated consecutive unselected primary elective unilateral THA and TKA between 1 February 2010 and 1 December 2011 in patients $\geq 18$ years with a Danish social security number and no prescriptions on 'potent' anticoagulant therapy (ie, vitamin $\mathrm{K}$ antagonists, dabigatran, rivaroxaban) $\leq 6$ months preoperatively. Procedures in patients with more than one THA or TKA during the study period were excluded if $<45$ days between operations.

Five departments participated throughout the study period, with a sixth department pausing between March 2010 and April 2011. All departments had a known mean LOS of about 3-4 days, with a similar fast-track setup including mobilisation on the day of surgery, identical functional discharge criteria and discharge to own home. ${ }^{19}$ Patients with the preoperative use of platelet inhibitors (acetylsalicylic acid, clopidogrel, dipyridamole, etc) ceased treatment 3-5 days prior to admission and resumed treatment the day after surgery. All patients completed a preoperative questionnaire on characteristics and comorbidity which was then entered into the Lundbeck Foundation Centre Database (LCDB ${ }^{20}$; see online supplementary appendix 1). Thromboprophylaxis was only given during hospitalisation in patients with LOS of $\leq 5$ days. If the LOS was $>5$ days, prophylaxis was prescribed by the attending surgeon according to local guidelines. The first dose of prophylaxis was given $6-8 \mathrm{~h}$ after surgery and consisted of either rivaroxaban (Xarelto, Bayer Pharma, Berlin, Germany) $10 \mathrm{mg} /$ day, enoxaparin (Klexane, Sanofi-Aventis, Paris, France) 4000 IU/day, dalteparin (Fragmin, Pfizer Health Care, New York, USA) 5000 IU/ day or fondaparinux (Arixtra, GlaxoSmithKline, London, UK) $2.5 \mathrm{mg}$ /day. No departments used IPCD. An interim analysis and a random-sample audit on treatment and data completion were conducted and approved by the steering committee in 2011 (see online supplementary appendix 2).

Preoperative data were cross-referenced with the Danish National Patient Registry (DNPR) regarding LOS and 90-day readmissions (including emergency room contacts, but excluding outpatient visits as clinical practice on treatment of TEE in Denmark includes an initial admission to hospital $^{21}$ ). LOS was defined as the number of postoperative nights in the hospital (including transferal to other departments) till discharge to the patients' own home. The DNPR registers all hospitalisations (including transferals, diagnoses and surgical procedures) at Danish hospitals, allowing information on the LOS and readmissions regardless of localisation. Reporting is mandatory for receiving reimbursement ensuring completeness of data of about $99.4 \% .^{22} 23$ To detect TEE during primary admission the complete medical records of patients with diagnosis codes related to TEE according to the International Classification of Diseases 10th revision, all transfers to other wards and the discharge summary of any patients with $\operatorname{LOS} \geq 5$ days were investigated. In case of readmission $\leq 90$ days, discharge files and/or patient files were investigated with regard to relation to surgery. ${ }^{20}$ The criteria for TEE were predefined as: DVT confirmed by ultrasound; PE confirmed by spiral CT; ventilation-perfusion scintigraphy or pathological removal of embolus and myocardial infarction (MI) with rise in biomarkers and ischaemic symptoms; diagnostic ECG changes; and primary coronary intervention or a coronary bypass graft. An ischaemic stroke was defined as a neurological symptom $>24 \mathrm{~h}$ and a positive CT scan, and a transient ischaemic attack was defined as a neurological symptom lasting less than $24 \mathrm{~h}$ and no new changes on CT scan. Mortality was obtained through the Central Office of Civil Registration using unique Danish social security numbers. The cause of death was obtained from the patient files/autopsies. In case of death outside the hospital with no autopsy, the patient's general practitioner was contacted regarding the cause of death. Adjudication of discharge summaries and patient files, apart from reasons for LOS $\geq 5$ days and death during admission, was blinded with regard to the duration of thrombosis prophylaxis. Adjudication was carried out by the first author (CCJ), and in case of possible TEE, the first author (CCJ) and senior author (HK) adjudicated cases together.

All prescriptions on 'potent' anticoagulant therapy and platelet inhibitors 6 months before and 3 months after surgery were investigated using The Danish National Database of Reimbursed Prescriptions (DNDRP). During the study period all prescriptions on 'potent' anticoagulant treatment received government reimbursements securing $100 \%$ completeness of data. ${ }^{24}$ Patients without prescriptions on anticoagulative therapy but answering 'yes' in the questionnaire were assumed to use platelet inhibitors. In 21 procedures in patients with LOS $\leq 5$ days we found only postoperative prescriptions on 'potent' anticoagulative therapy. All hospital contacts of these 
patients were reviewed and, if insufficient for determining the cause of the prescription, we contacted their general practitioner. In two cases the prescription was due to DVT found during outpatient visits without regular hospital admission, while seven cases were due to perioperative atrial-flutter with treatment initiated $\geq 22$ days after discharge. These were all retained in the study cohort. The remaining 12 cases were due to specific surgical considerations, discharge from other wards or readmission with treatment despite unverified VTE. These were considered protocol violations and included in the unsuccessful early discharge cohort.

\section{Outcomes}

The primary outcome was the occurrence of symptomatic TEE (DVT, PE, arterial embolism, MI, ischaemic stroke or TCA) and VTE (DVT or PE) 90 days after THA/TKA in patients with prophylaxis only during admission.

The secondary outcome was the occurrence of the primary outcome in patients with thromboprophylaxis after discharge. Bleeding events were protocolled as a safety endpoint, but were hindered by incomplete registration. A separate analysis on patients not in the LCDB was carried out to identify potential bias.

\section{Statistics and power calculation}

A prestudy power analysis using a two-tailed one sample difference from constant test, found that 2838 patients were needed to detect a $1 \%$ increase in TEE when assuming a TEE rate of $3 \%, \beta: 82$ and $\alpha: 0.05$. Correspondingly, 2076 patients were needed to detect a $1 \%$ increase in symptomatic VTE assuming a 90-day baseline risk of $2 \%{ }^{2}$

Data were tested for normality using q-q plots and histograms. Comparisons of continuous data were made using Mann-Whitney $\mathrm{U}$ test and $\mathrm{t}$ test and for categorical data with $\chi^{2}$ test or Fisher's exact test, as appropriate. Events (incident cases) are reported as actual numbers and percentages with $95 \%$ CI using http://www.vassarstats.net/ prop1.html. All other analysis was carried out in SPSS V.20 (IBM Corporation, Armonk, New York, USA).

\section{RESULTS}

Total cohort

A total of 4924 procedures in 4718 patients were included. The median LOS was 2 days (IQR 2-3), and readmissions occurred after $400(8.1 \%)$ of all procedures. We found $50(1.12 \%)$ TEE, of which $30(0.58 \%)$ were VTE. Symptomatic in-hospital TEE occurred after 7 $(0.14 \%(0.07 \%$ to $0.29 \%))$ procedures, of which 4 $(0.08 \%(0.03 \%$ to $0.21 \%))$ were VTE. All-cause mortality was $0.42 \%$, including one fatal PE $(0.04 \%)$ and six deaths of unknown causes $(0.1 \%$; table 2$)$.

\section{Successful early discharge cohort}

Early discharge with LOS $\leq 5$ days occurred in 4659 $(94.6 \%)$ procedures in 4455 patients (figure 1). These patients had a mean age of 66.8 years (SD 10.7) with a median LOS and prophylaxis duration of 2 days (IQR 2-3; table 1). There were 353 (7.6\%) surgery-related readmissions of which $2.9 \%$ were due to 'surgical' morbidity (hip displacements, prosthesis infections, knee manipulation, etc) and $4.7 \%$ were due to 'medical' morbidity, such as anaemia, cardiac arrhythmia, pneumonia, unverified prosthesis infection and pain.

A total of $39(0.84 \%)$ TEE were found within 90 days, of which $24(0.52 \%, 95 \%$ CI $0.35 \%$ to $0.77 \%)$ occurred during the first postoperative month. One patient was readmitted twice due to ischaemic strokes on postoperative days 8 and 46. According to the medical records, the second stroke was cardiac in origin as the patient was known to have an atrial flutter, but treated only with acetylsalicylic acid due to gastrointestinal bleeding. There were $19(0.41 \%)$ symptomatic VTE (figures 2 and $3 \mathrm{~A}$ ), consisting of $5(0.11 \%)$ PEs and 14 $(0.30 \%)$ DVTs of which 9 were proximal (table 2). Median time to VTE was 21 days (IQR 8-39), with 12 VTE $\leq 30$ days postoperatively (30-day VTE rate $0.26 \%$, $95 \%$ CI $0.15 \%$ to $0.45 \%)$.

There were $13(0.28 \%)$ deaths during follow-up. Of these three $(0.06 \%)$ were unrelated to surgery (cancer and gastric morbidity $>45$ days after surgery) and six $(0.13 \%)$ were of unknown causes outside hospital (postoperative day: 19, 27, 36, 44, 48 and 85). Thus, four $(0.09 \%)$ deaths were confirmed surgically related (table 2), one due to an autopsy confirmed PE on postoperative day 41 and one due to intracerebral bleeding on day 26. The remaining two deaths were due to paralytic ileus on postoperative day 36 and sepsis on postoperative day 24.

Unsuccessful early discharge cohort: This cohort of 265 $(5.4 \%)$ procedures in 263 patients (figure 1), was older and had more comorbidity than the early discharge cohort (table 1). The median LOS was 7 days (IQR 6-9) with $47(17.7 \%)$ surgically related readmissions $(5.7 \%$ 'surgical' and 12\% 'medical' morbidity). Of 11 (4.97\%) TEE with 7 (2.65\%) VTE (table 2), 7 and 4, respectively, occurred during index hospitalisation consequently resulting in LOS $>5$ days. Of the four $(1.51 \% \quad(95 \% \mathrm{CI}$ $0.59 \%$ to $3.82 \%)$ ) TEE after discharge, one $(0.38 \%$ $(0.07 \%$ to $2.11 \%))$ was an ischaemic stroke and three were VTE $(1.13 \%(0.38 \%$ to $3.27 \%))$, with two PEs $(0.75 \%(0.21 \%$ to $2.70 \%))$ and one DVT $(0.38 \%(0.07 \%$ to $2.11 \%$ )). Median time to VTE was 3 days (IQR 2-53; figure $3 \mathrm{~B})$. We found three $(1.13 \%)$ surgically related deaths, one death unrelated to surgery (paralytic ileus on day 70 in a patient refusing treatment) and no VTE-related deaths or deaths of unknown causes $(0.00 \%$ $(0.00 \%$ to $1.43 \%))$.

\section{Patients not in LCDB (3.6\%)}

In these 194 (108 THA/86 TKA) procedures in 191 patients, the mean age was 68.5 years (SD 11.0) and 178 $(91.8 \%)$ had LOS $\leq 5$ days. In these 178 procedures there was one $(0.56 \%(0.10 \%$ to $3.11 \%))$ readmission due to an MI, no VTE and no deaths. No further 
Figure 1 Flow chart of the study population. THA, total hip arthroplasty; TKA, total knee arthroplasty.

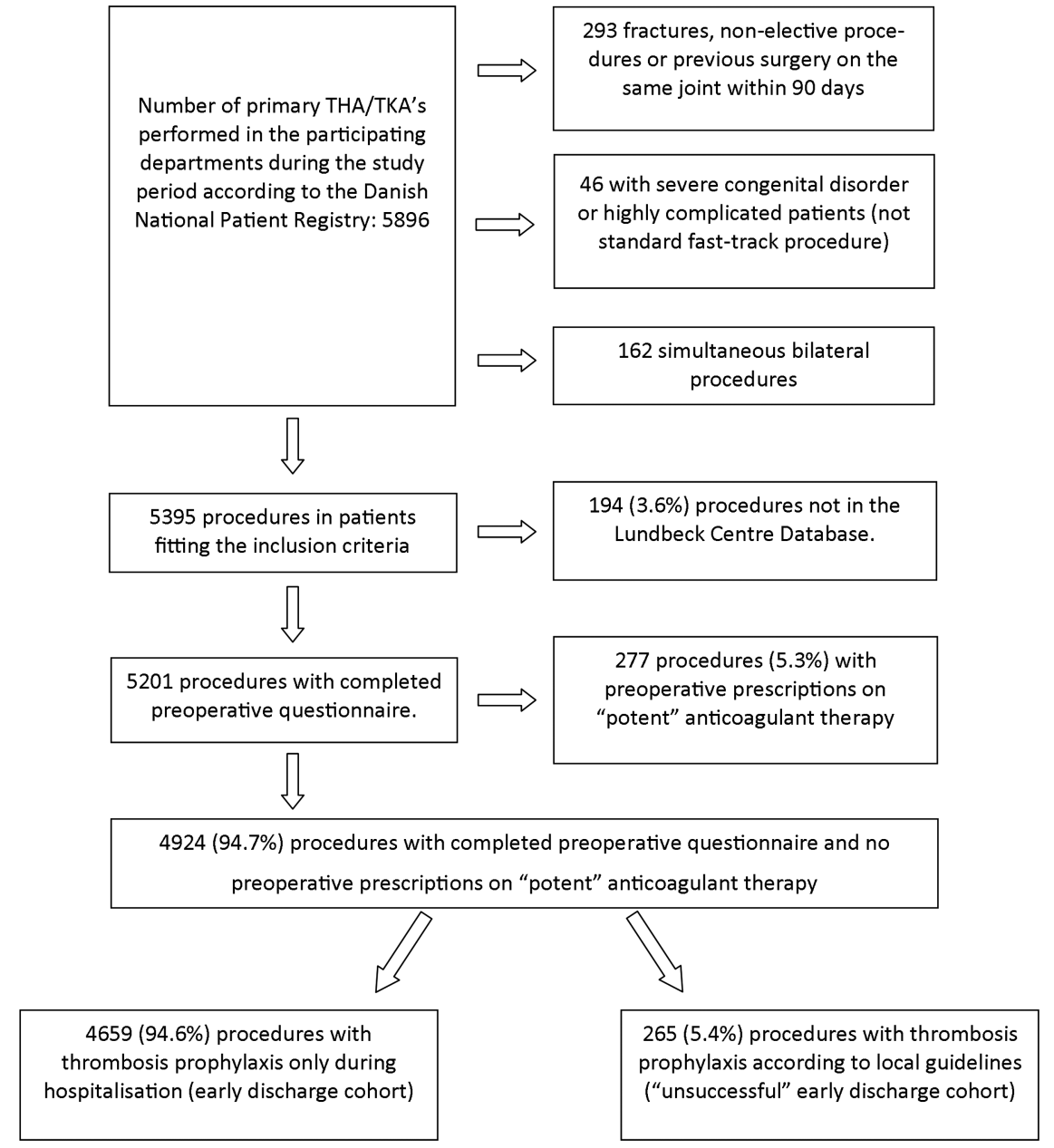

analysis was carried out as no bias was apparent compared to the study population.

\section{DISCUSSION}

In this prospective study in fast-track primary THAs and TKAs, we found 90-day postoperative rates of symptomatic TEE and VTE of $0.84 \%$ and $0.41 \%$, respectively, in patients with LOS $\leq 5$ days and in-hospital thromboprophylaxis only. The patients receiving prophylaxis only during index hospitalisation (median 2 days) contributed $94.6 \%$ of the total number of performed procedures, as $5.4 \%$ had LOS $>5$ days and consequently received longer prophylaxis. The study has several strengths, such as a consecutive unselected population including high-risk patients with various types of comorbidity, a standardised perioperative fast-track setup and complete detailed 90-day follow-up.

We used any TEE as primary endpoint in order not to overlook a potential worsened outcome. Stroke and MI have been included as safety endpoints in most RCTs, ${ }^{10-12}$ but are often neglected in reviews and database studies. ${ }^{25-27}$ We found no increase in the occurrence of ischaemic stroke as compared with previous studies of in-hospital stroke in $\mathrm{THA}^{28}$ and strokes $\leq 30$ days in both TKA and THA, ${ }^{29}$ despite our follow-up being 90 days and not relying on diagnostic codes. Neither was there any apparent increase in the occurrence of MI compared with a recent study which found MI in $0.51 \%$ of THA and $0.21 \%$ of TKA after 6 weeks. ${ }^{30}$ The numbers of symptomatic VTE were lower or comparable to the RCTs with prophylaxis of 1035 days. ${ }^{10-13}$ However, LOS in these RCTs was 8-12 days with unspecified discharge locations, whereas LOS after $94.6 \%$ of procedures in our study was $\leq 5$ days until discharge to own home. The long LOS in these studies may include partial immobilisation, thereby increasing the risk of VTE and consequently the need for thromboprophylaxis. Correspondingly, a previous small-scale study in 247 TKA found a decreased risk of DVT following mobilisation within $24 \mathrm{~h}$ of surgery, ${ }^{14}$ and an earlier fast-track singlecentre study with prophylaxis only during admission in 1977 THA and TKAs with a mean LOS of about 3.5 days found $0.86 \%$ symptomatic VTE within 90 days. ${ }^{18}$

Another main difference between our study and the RCTs is that there was no preoperative selection of patients, as the duration of prophylaxis depended only on discharge within 5 days, regardless of comorbidity. Thus, our results reflect 'everyday patients', whereas the exclusion criteria in the RCTs may have reduced occurrences of TEE. ${ }^{8} 31$ The only excluded patients in our study were those using preoperative 'potent' anticoagulant therapy, since they obviously needed continuation 
Table 1 Preoperative patient characteristics and prophylaxis duration

\begin{tabular}{|c|c|c|c|c|c|c|c|}
\hline Characteristic & $\begin{array}{l}\text { Early } \\
\text { discharge } \\
\mathrm{N}: 4659 \\
\end{array}$ & $\begin{array}{l}\text { 'Unsuccessful' } \\
\text { early discharge } \\
\mathrm{N}: 265\end{array}$ & p Value & Characteristic & $\begin{array}{l}\text { Early } \\
\text { discharge } \\
\mathrm{N}: 4659 \\
\end{array}$ & $\begin{array}{l}\text { 'Unsuccessful' } \\
\text { early discharge } \\
\mathrm{N}: 265\end{array}$ & p Value \\
\hline Age (SD) (years) & $66.8(10.7)$ & $73.0(12.1)$ & $<0.001$ & BMI (SD) & $28.4(5.1)$ & $27.9(5.7)$ & 0.110 \\
\hline$<50$ & $313(6.7)$ & $11(4.2)$ & & $<18.5$ & $35(0.8)$ & $5(1.9)$ & \\
\hline $50-60$ & 855 (18.4) & $28(10.6)$ & & $18.5-24.9$ & $1186(25.6)$ & 79 (30.4) & \\
\hline $61-65$ & 779 (16.7) & 19 (7.2) & & $25.0-29.9$ & 1865 (40.2) & $102(39.2)$ & \\
\hline $66-70$ & 916 (19.7) & $34(12.8)$ & & $30.0-39.9$ & $1426(30.7)$ & $63(26.0)$ & \\
\hline $71-75$ & $807(17.3)$ & $47(17.7)$ & & $\geq 40$ & $126(2.7)$ & $11(3.7)$ & \\
\hline $76-80$ & 585 (12.6) & $50(18.9)$ & & Missing & $21(0.5)$ & $5(1.9)$ & \\
\hline $81-86$ & $302(6.5)$ & $45(17.0)$ & & & & & \\
\hline$>86$ & $102(2.2)$ & $31(11.7)$ & & & & & \\
\hline Gender & & & 0.002 & Joint & & & 0.961 \\
\hline Females & $2654(57.0)$ & $177(66.8)$ & & THA & 2451 (52.6) & 139 (52.5) & \\
\hline Males & $2005(43.0)$ & $88(33.2)$ & & TKA & $2208(47.4)$ & $126(47.5)$ & \\
\hline Use of compressive & & & $<0.001$ & Diabetes & & & 0.426 \\
\hline stockings & & & & T1D & $14(0.3)$ & $2(0.7)$ & \\
\hline Yes & $250(5.5)$ & 35 (13.7) & & T2D & 505 (10.9) & $30(11.5)$ & \\
\hline No & 4267 (94.5) & $220(86.3)$ & & None & $4112(88.8)$ & $230(87.8)$ & \\
\hline Missing & $142(3.0)$ & $10(3.8)$ & & Missing & $28(0.6)$ & $3(1.1)$ & \\
\hline Social situation & & & $<0.001$ & Hypertension & & & $<0.001$ \\
\hline Living with others & 3117 (66.9) & $117(44.2)$ & & Yes & 2291 (49.5) & $161(61.2)$ & \\
\hline Living alone & 1502 (32.2) & $139(52.5)$ & & No & 2335 (50.5) & $102(38.8)$ & \\
\hline Nursing home, etc & $40(0.9)$ & $9(3.4)$ & & Missing & $33(0.7)$ & $2(0.8)$ & \\
\hline Use of walking aid & $1078(23.7)$ & $142(55.0)$ & $<0.001$ & $\begin{array}{l}\text { Pharmacologically } \\
\text { treated PsD }\end{array}$ & & & $<0.001$ \\
\hline Yes & 3469 (76.3) & $116(45.0)$ & & Yes & $311(6.7)$ & $33(12.6)$ & \\
\hline No & $112(2.4)$ & 7 (2.6) & & No & 4308 (93.3) & $228(87.4)$ & \\
\hline Missing & & & & Missing & $40(0.9)$ & $4(1.5)$ & \\
\hline Hypercholesterolaemia & & & 0.044 & Prior cerebral stroke & & & $<0.001$ \\
\hline Yes & $1289(28.0)$ & 89 (33.7) & & & & & \\
\hline No & 3321 (72.0) & $175(66.3)$ & & Yes & $250(5.5)$ & $29(11.2)$ & \\
\hline Missing & $49(1.1)$ & $1(0.4)$ & & No & $4336(94.5)$ & $229(88.8)$ & \\
\hline & & & & Missing & $73(1.6)$ & $7(2.6)$ & \\
\hline Smoking & & & 0.058 & Prior VTE & & & $<0.001$ \\
\hline Yes & 703 (15.2) & $51(19.2)$ & & Yes & $179(3.9)$ & $22(8.5)$ & \\
\hline No & 3908 (84.8) & $209(80.4)$ & & No & 4401 (96.1.0) & 261 (91.5) & \\
\hline Missing & $48(1.0)$ & $5(1.9)$ & & Missing & $79(1.7)$ & $6(2.3)$ & \\
\hline Alcohol >2 units daily & & & 0.015 & Relative with VTE & & & 0.023 \\
\hline Yes & $345(7.5)$ & $9(3.4)$ & & Yes & 507 (12.2) & $16(7.1)$ & \\
\hline No & $4263(91.5)$ & $252(96.6)$ & & No & $3643(87.8)$ & 208 (92.9) & \\
\hline Missing & $51(1.1)$ & $4(1.5)$ & & Missing & 509 (10.9) & $41(15.5)$ & \\
\hline $\begin{array}{l}\text { Pharmacologically } \\
\text { treated PD }\end{array}$ & & & 0.094 & $\begin{array}{l}\text { Anticoagulative } \\
\text { treatment }\end{array}$ & & & $<0.001$ \\
\hline Yes & $333(7.2)$ & $26(10.0)$ & & Platelet inhibitors & $1284(26.2)$ & $120(39.7)$ & \\
\hline No & $4286(92.8)$ & $264(90.0)$ & & None & 3375 (68.9) & $145(48.0)$ & \\
\hline Missing & $44(0.9)$ & $5(1.9)$ & & Missing & $0(0)$ & $0(0)$ & \\
\hline $\begin{array}{l}\text { Pharmacologically } \\
\text { treated CD }\end{array}$ & & & 0.005 & $\begin{array}{l}\text { Duration of } \\
\text { prophylaxis }\end{array}$ & & & \\
\hline Yes & $418(9.1)$ & $37(14.4)$ & & Mean (SD) & $2.5(0.91)$ & $N / A$ & \\
\hline No & 4175 (90.9) & 220 (85.6) & & Median (IQR) & $2(2-3)$ & $\mathrm{N} / \mathrm{A}$ & \\
\hline Missing & 66 (1.4) & $8(3.0)$ & & & & & \\
\hline
\end{tabular}

Data reported as $n(\%)$ for counts and mean for continuous variables unless otherwise specified.

BMI, body mass index; CD, cardiac disease; LOS, length of hospital stay; N, procedures; N/A, not available; PD, pulmonary disease;

PsD, psychiatric disease; T1D, type 1 diabetes; T2D, type 2 diabetes; THA, total hip arthroplasty; TKA, total knee arthroplasty; VTE, venous thromboembolic event.

after discharge. Two Danish nationwide studies found symptomatic VTE in $>1 \%$ of THA and TKA despite prolonged prophylaxis, and that the incidence was increasing across the study periods (1995-2007). ${ }^{26}{ }^{32}$ The difference between these data and ours may be due to the fast-track set-up including early mobilisation in 


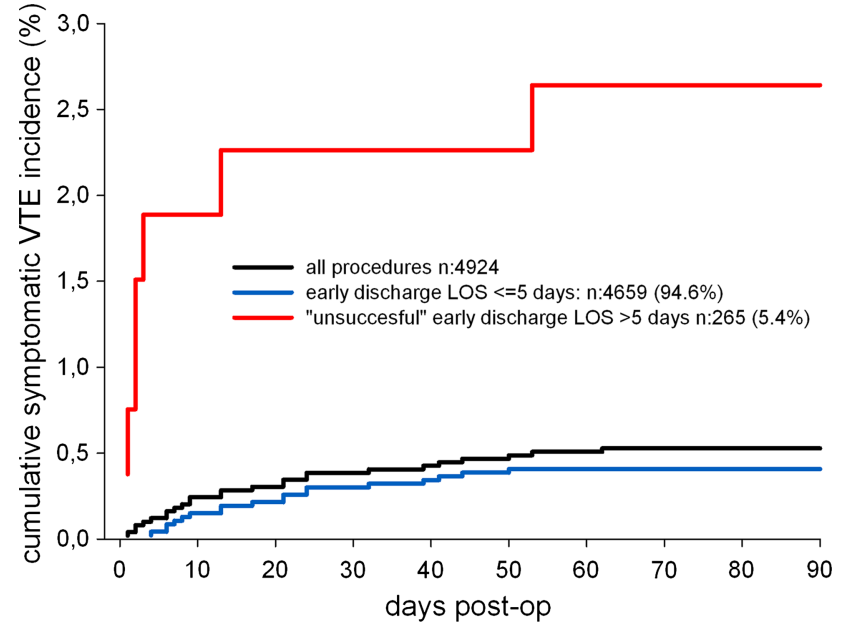

Figure 2 Cumulated incidence of symptomatic venous thromboembolic events.

our study, and since LOS in Denmark was about 11 days in year $2000 .^{33}$

The occurrence of in-hospital TEE in the total 4924 procedures in the LCDB was low $(0.14 \%)$, and particularly the incidence of symptomatic in-hospital VTE $(<0.10 \%)$ was lower than the $0.5 \%$ in THA and $1 \%$ in TKA found in a recent review. ${ }^{25}$ Although the timing of
VTE, with the majority occurring within the first month is consistent with previous studies, ${ }^{2}{ }^{34}$ we believe that the low incidence questions the benefits of prolonged prophylaxis in all patients after fast-track THA and TKA. Further studies are needed to identify whether certain patient subgroups may benefit from more extensive or intensive prophylaxis, and how to avoid in-hospital TEE while patients are receiving recommended treatment. However, due to the few events the numbers of patients needed for such studies pose major challenges.

Finally, we report both confirmed VTE-related death and a 'worst case' scenario, with death of unknown causes being considered VTE-related, despite that cause of death after THA/TKA often is found unrelated to VTE. ${ }^{35}$ Thus, we found only one verified fatal PE, and a 90-day all-cause mortality comparable to or lower than previous studies. $^{27} 36-38$

The 'unsuccessful' early discharge cohort was older with more comorbidity and readmissions. This is not surprising, as we have previously found an association with LOS and readmissions after fast-track THA and TKA in such patients. ${ }^{20}$ There were about $2 \%$ PEs in these patients, but this is in accordance with comorbidities such as cardiac disease or previous TEE, being associated with cardiac and thromboembolic complications after arthroplasty. ${ }^{27}$ Furthermore, complications per se
Figure 3 (A and B) Timing of type of TEE in the early discharge cohort $(A)$ and the 'unsuccessful' early discharge cohort (B). TEE, thromboembolic event; VTE, venous thromboembolic event. Dotted line marks postoperative day 30 .

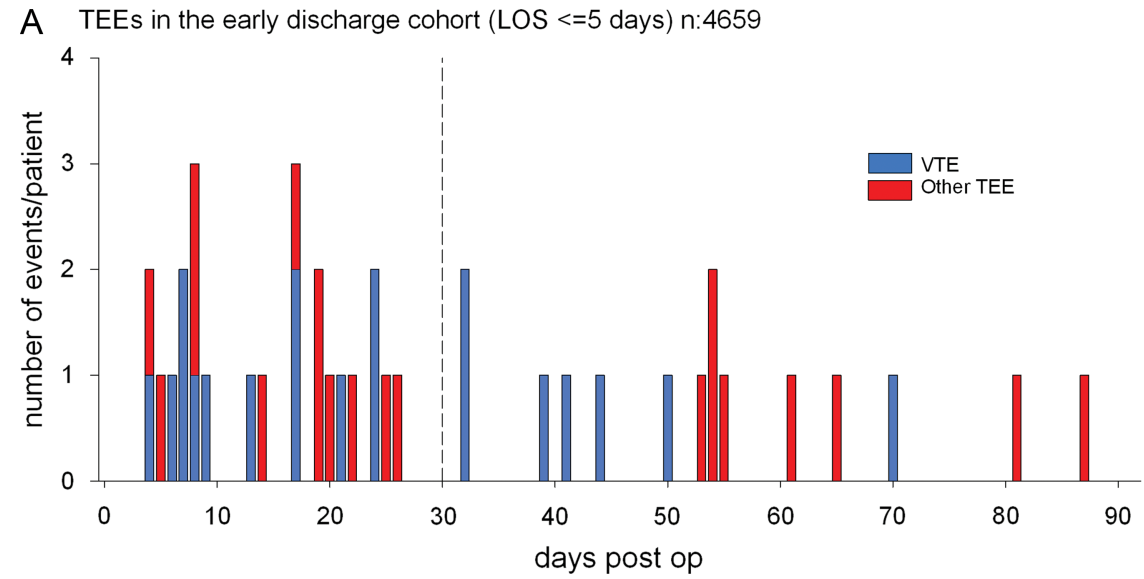

B TEEs in the "unsuccessful" cohort (LOS $>5$ days) n:265

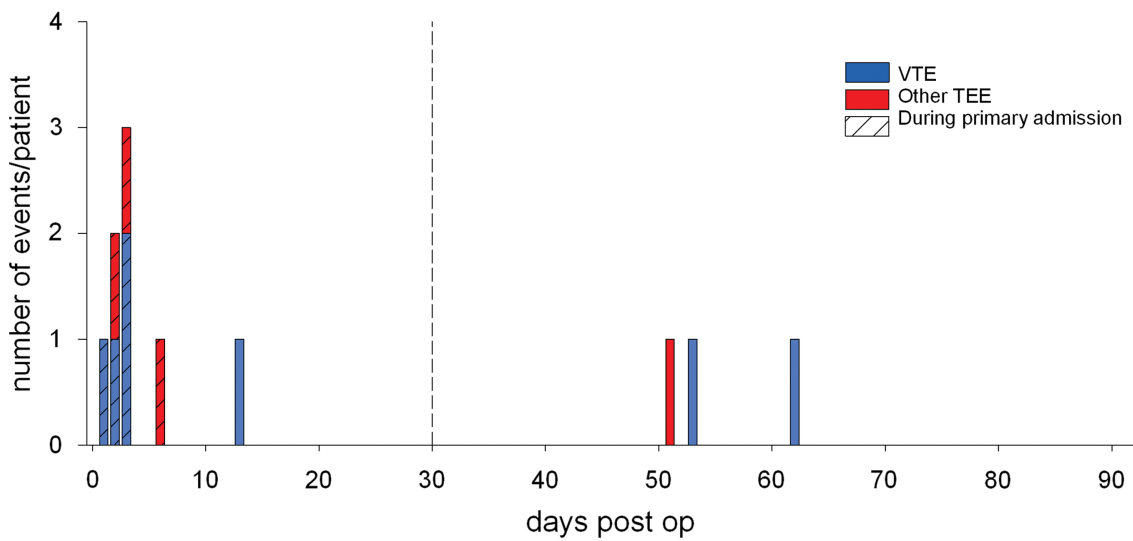


Table 2 VTEs, all TEEs and mortality

\begin{tabular}{|c|c|c|c|}
\hline Outcomes & All procedures (n: 4924) & Early discharge (n: 4659) & $\begin{array}{l}\text { 'Unsuccessful' early } \\
\text { discharge (n: 265) }\end{array}$ \\
\hline PE & $10(0.21 ;(0.12$ to 0.38$))$ & $5(0.11 ;(0.05$ to 0.25$))$ & 5 (1.99; (0.92 to 4.27$))$ \\
\hline Any DVT & $16(0.37 ;(0.24$ to 0.58$))$ & $14(0.30 ;(0.18$ to 0.50$))$ & $2(0.67 ;(0.18$ to 2.38$))$ \\
\hline Proximal DVT & $11(0.23 ;(0.13$ to 0.41$))$ & $9(0.19 ;(0.10$ to 0.36$))$ & $2(0.67 ;(0.18$ to 2.38$))$ \\
\hline Any VTE & $30(0.58 ;(0.41$ to 0.83$))$ & $19(0.41 ;(0.26$ to 0.64$))$ & 7 (2.65; (1.35 to 5.14$))$ \\
\hline Any VTE (THA/TKA) & $\begin{array}{l}17 / 13(0.62 ;(0.39 \text { to } 0.99)) / \\
(0.53 ;(0.31 \text { to } 0.90))\end{array}$ & $\begin{array}{l}15 / 4(0.61 ;(0.37 \text { to } 1.00)) / \\
(0.18 ;(0.07 \text { to } 0.46))\end{array}$ & $\begin{array}{l}1 / 6(0.65 ;(0.11 \text { to } 3.60)) / \\
(4.70 ;(2.31 \text { to } 9.38))\end{array}$ \\
\hline Myocardial infarction & $8(0.17 ;(0.09$ to 0.32$))$ & $7(0.15 ;(0.07$ to 0.31$))$ & 1 (0.33; (0.06 to 1.85$))$ \\
\hline Ischaemic stroke & $8(0.19 ;(0.10$ to 0.35$))$ & $6(0.13 ;(0.06$ to 0.28$))$ & $2(0.99 ;(0.34$ to 2.87$))$ \\
\hline Transient ischaemic attack & $7(0.15 ;(0.08$ to 0.30$))$ & $7(0.15 ;(0.07$ to 0.31$))$ & $0(0.33 ;(0.06$ to 1.85$))$ \\
\hline Arterial embolus & $1(0.04 ;(0.01$ to 0.14$))$ & $0(0.00 ;(0.00$ to 0.08$))$ & 1 (0.66; (0.18 to 2.38)) \\
\hline Any TEE & $50(1.12 ;(0.87$ to 1.44$))$ & $39(0.84 ;(0.62$ to 1.15$))$ & $11(4.97 ;(3.04$ to 8.04$))$ \\
\hline Any TEE (THA/TKA) & $\begin{array}{l}29 / 21(1.17 ;(0.83 \text { to } 1.65)) / \\
(1.05 ;(0.72 \text { to } 1.53))\end{array}$ & $\begin{array}{l}27 / 12(1.10 ;(0.76 \text { to } 1.60)) / \\
(0.54 ;(0.31 \text { to } 0.94))\end{array}$ & $\begin{array}{l}2 / 9(1.96 ;(0.67 \text { to } 5.60)) / \\
(8.05 ;(4.66 \text { to } 13.54))\end{array}$ \\
\hline All-cause mortality & $17(0.42 ;(0.28$ to 0.64$))$ & $13(0.28 ;(0.16$ to 0.49$))$ & 4 (1.99; (0.92 to 4.27$))$ \\
\hline Unrelated to surgery & $4(0.10 ;(0.04$ to 0.23$))$ & 3 (0.06; (0.01 to 0.20$))$ & $1(0.33 ;(0.06$ to 1.85$))$ \\
\hline Surgically related mortality & $7(0.19 ;(0.10$ to 0.35$))$ & $4(0.09 ;(0.04$ to 0.23$))$ & 3 (1.66; (0.71 to 3.82$))$ \\
\hline Death of unknown cause & $6(0.13 ;(0.06$ to 0.27$))$ & $6(0.13 ;(0.06$ to 0.28$))$ & $0(0.00 ;(0.00$ to 1.26$))$ \\
\hline Fatal PE & $1(0.04 ;(0.01$ to 0.14$))$ & $1(0.02 ;(0.00$ to 0.12$))$ & $0(0.33 ;(0.06$ to 1.85$))$ \\
\hline Fatal PE/death of unknown cause & $7(0.17 ;(0.09$ to 0.32$))$ & $7(0.15 ;(0.07$ to 0.31$))$ & $0(0.33 ;(0.06$ to 1.85$))$ \\
\hline Any VTE or death of unknown cause & $32(0.71 ;(0.52$ to 0.98$))$ & $25(0.54 ;(0.37$ to 0.80$))$ & $7(2.65 ;(1.35$ to 5.14$))$ \\
\hline Any TEE or death of unknown cause & 56 (1.25; (0.98 to 1.59$))$ & $45(0.97 ;(0.73$ to 1.29$))$ & $11(4.97 ;(3.04$ to 8.04$))$ \\
\hline
\end{tabular}

may lead to prolonged LOS and thereby longer prophylaxis. Thus, about $60 \%$ of TEE and VTE in this cohort occurred during primary admission. However, it does not argue against our conclusion that prophylaxis only during admission is safe when LOS $\leq 5$ days.

Our study has limitations, foremost regarding the follow-up which was based on hospital contacts. However, although the reliability of diagnostic codes for VTE in DNPR may be low, ${ }^{39}$ the completion of data regarding somatic admissions is close to $100 \% .^{22}{ }^{39}$ Consequently, we investigated all admissions through discharge summaries and patient files instead of relying only on diagnostic codes, as often carried out in large-scale cohort studies. ${ }^{26} 3240$ Although TEE may have been left out of the discharge summary, this seems unlikely, as they require treatment after discharge. We also used the DNDRP to detect procedures followed by a postoperative prescription of potent anticoagulant therapy, thereby ensuring that any TEE diagnosed in outpatient clinics would be registered. The DNDRP is ideally suited for this, as all prescriptions on oral anticoagulants in Denmark receive reimbursement and are therefore recorded. Regarding TEE during primary admission, ideally we should have investigated the discharge summaries of every procedure with LOS $\leq 5$ days. However, as TEEs are serious complications they would require prolonged hospitalisation. Thus, LOS in all seven patients with TEE during primary admission was $>5$ days (figure $3 \mathrm{~B}$ ).

The local guidelines for thrombosis prophylaxis in the participating departments were 6-10 days after discharge when LOS $>5$ days, and therefore it may be problematic that we do not have exact data on the duration of prophylaxis for the secondary cohort. However, this does not change the conclusion-that prophylaxis only during admission is safe in THA and TKA with LOS $\leq 5$ days. It could also be argued that our study should have been carried out as an RCT. However we did not attempt to compare two types of treatment. Instead, for complex medical situations detailed cohort studies have been proposed as a viable, and sometimes preferable, alternative. ${ }^{41} 42$ In this context, a post hoc analysis assuming a $2 \%$ baseline risk of symptomatic VTE with extended LMWH prophylaxis of about 35 days $^{2}$ found the actual power of our study to be $99 \%$ due to the large number of patients. Whether our cut-off of 5 days LOS is an optimal way of deciding on duration of prophylaxis is uncertain, but it seems unlikely that patients with a satisfactory fasttrack procedure would have a longer LOS. ${ }^{33}$ However, it is worth noticing that $>75 \%$ of procedures were followed by LOS, and consequently thromboprophylaxis, for $\leq 3$ days and that about $95 \%$ of all procedures had LOS $\leq 5$ days.

In conclusion, we found low rates of TEE and VTE after primary elective fast-track THA and TKA with thromboprophylaxis only during hospitalisation in patients with LOS $\leq 5$ days. These results support previous findings from other types of surgery, suggesting that guidelines on postoperative thromboprophylaxis need reconsideration in modern elective surgical procedures.

\section{Author affiliations}

${ }^{1}$ Section for Surgical Pathophysiology, The Juliane Marie Centre, Rigshospitalet, Copenhagen University, Copenhagen, Denmark 
2Department of Orthopaedics, Aarhus University Hospital, Aarhus University, Aarhus N, Denmark

${ }^{3}$ Department of Orthopaedics, Regional Hospital Holstebro, Aarhus University, Holstebro, Denmark

${ }^{4}$ Orthopaedic Department, Hvidovre University Hospital, Hvidovre, Denmark

${ }^{5}$ Department of Orthopedics, Vejle Hospital, University of Southern Denmark, Vejle, Denmark

${ }^{6}$ Orthopaedic Department, Sydvestjysk Hospital Esbjerg/Grindsted, Grindsted, Denmark

${ }^{7}$ Orthopaedic Division, Aalborg University Hospital Northern, Aalborg University, Farsø, Denmark

Acknowledgements The authors would like to thank Professor Lars Pedersen (Department of Clinical Epidemiology Aarhus University Hospital), for help with data extraction from the Danish National Database of Reimbursed Prescriptions, which is supported by a grant from the Clinical Epidemiological Research Foundation and Aarhus University.

Contributions CCJ updated the initial protocol, registered the trial, undertook all data gathering, performed all statistical analyses, produced all tables and figure, wrote the first manuscript draft, revised it and submitted it for publication. MKJ wrote the initial protocol, helped implement the study setup and helped revise the manuscript. KS supervised the initial protocol, implemented the study setup at Aarhus hospital, conducted the interim analysis and randomised sample audit and helped revising the manuscript. TBH helped develop the initial protocol, implemented the study setup at regional hospital Holstebro, conducted the randomised sample audit and helped revising the manuscript. HK supervised the initial protocol, supervised the work carried out by CCJ, contributed to data analysis and helped to draft and revise the manuscript. HH, PK-A, LTH and MBL helped develop the initial protocol, implemented the study setup at their respective study locations and revised the manuscript. All authors approved of the final version to be published. CCJ had full access to all of the data in the study and takes responsibility for the integrity of the data and the accuracy of the data analysis.

Funding The study was supported by a grant from the Lundbeck Foundation. The Lundbeck Foundation had no influence on any part of the study or on the development of the manuscript.

Competing interests $\mathrm{HH}$ and $\mathrm{HK}$ are board members of the Health Care initiatives, Biomet Rapid Recovery.

Ethics approval The Regional Ethics Committee waived the need for study approval. Permission was acquired from the Danish National Board of Health j. nr:3-3013-56/1/HKR and the Danish Data Protection Agency j.nr: 2007-58-0015 to review and store patient records without informed consent.

Provenance and peer review Not commissioned; externally peer reviewed.

Data sharing statement Patient-level data and full dataset available from the corresponding author. Consent was not obtained but the presented data are anonymised and risk of identification is low.

Open Access This is an Open Access article distributed in accordance with the Creative Commons Attribution Non Commercial (CC BY-NC 3.0) license, which permits others to distribute, remix, adapt, build upon this work noncommercially, and license their derivative works on different terms, provided the original work is properly cited and the use is non-commercial. See: http://creativecommons.org/licenses/by-nc/3.0/

\section{REFERENCES}

1. Raskob GE, Silverstein R, Bratzler DW, et al. Surveillance for deep vein thrombosis and pulmonary embolism: recommendations from a national workshop. Am J Prev Med 2010;38:S502-9.

2. Falck-Ytter Y, Francis CW, Johanson NA, et al. Prevention of VTE in orthopedic surgery patients: Antithrombotic Therapy and Prevention of Thrombosis, 9th ed: American College of Chest Physicians Evidence-Based Clinical Practice Guidelines. Chest 2012;141: e278S-325S.

3. Gould MK, Garcia DA, Wren SM, et al. Prevention of VTE in nonorthopedic surgical patients: Antithrombotic Therapy and Prevention of Thrombosis, 9th ed: American College of Chest
Physicians Evidence-Based Clinical Practice Guidelines. Chest 2012;141:e227S-7S.

4. NICE guidelines 2010 Venous thromboembolism: reducing the risk. http://www.nice.org.uk/nicemedia/live/12695/47195/47195.pdf (accessed 8-7-2013).

5. Qadan M, Polk HC Jr, Hohmann SF, et al. A reassessment of needs and practice patterns in pharmacologic prophylaxis of venous thromboembolism following elective major surgery. Ann Surg 2011;253:215-20.

6. Kjaersgaard-Andersen $\mathrm{P}$, Kehlet $\mathrm{H}$. Should deep venous thrombosis prophylaxis be used in fast-track hip and knee replacement? Acta Orthop 2012;83:105-6.

7. Jameson SS, Bottle A, Malviya A, et al. The impact of national guidelines for the prophylaxis of venous thromboembolism on the complications of arthroplasty of the lower limb. J Bone Joint Surg Br 2010;92:123-9.

8. Mont MA, Jacobs JJ, Boggio LN, et al. Preventing venous thromboembolic disease in patients undergoing elective hip and knee arthroplasty. J Am Acad Orthop Surg 2011;19:768-76.

9. Eriksson BI, Borris LC, Friedman RJ, et al. Rivaroxaban versus enoxaparin for thromboprophylaxis after hip arthroplasty. $N$ Engl J Med 2008;358:2765-75.

10. Lassen MR, Gallus A, Raskob GE, et al. Apixaban versus enoxaparin for thromboprophylaxis after hip replacement. $N$ Engl $J$ Med 2010;363:2487-98.

11. Lassen MR, Raskob GE, Gallus A, et al. Apixaban versus enoxaparin for thromboprophylaxis after knee replacement (ADVANCE-2): a randomised double-blind trial. Lancet 2010;375:807-15

12. Turpie AG, Lassen MR, Davidson BL, et al. Rivaroxaban versus enoxaparin for thromboprophylaxis after total knee arthroplasty (RECORD4): a randomised trial. Lancet 2009;373:1673-80.

13. Kakkar AK, Brenner B, Dahl OE, et al. Extended duration rivaroxaban versus short-term enoxaparin for the prevention of venous thromboembolism after total hip arthroplasty: a double-blind, randomised controlled trial. Lancet 2008;372:31-9.

14. Pearse EO, Caldwell BF, Lockwood RJ, et al. Early mobilisation after conventional knee replacement may reduce the risk of postoperative venous thromboembolism. J Bone Joint Surg $\mathrm{Br}$ 2007;89:316-22.

15. Ansari D, Gianotti L, Schroder J, et al. Fast-track surgery: procedure-specific aspects and future direction. Langenbecks Arch Surg 2013;398:29-37.

16. Kehlet H. Fast-track hip and knee arthroplasty. Lancet 2013;381:1600-2

17. Qadan M, Tyson M, McCafferty MH, et al. Venous thromboembolism in elective operations: balancing the choices. Surgery 2008;144:654-60, discussion.

18. Husted $\mathrm{H}$, Otte KS, Kristensen BB, et al. Low risk of thromboembolic complications after fast-track hip and knee arthroplasty. Acta Orthop 2010;81:599-605.

19. Husted H, Solgaard S, Hansen TB, et al. Care principles at four fast-track arthroplasty departments in Denmark. Dan Med Bull 2010;57:A4166.

20. Jorgensen $\mathrm{CC}$, Kehlet $\mathrm{H}$. Role of patient characteristics for fast-track hip and knee arthroplasty. Br J Anaesth 2013;110:972-80.

21. Handbook of emergency medicine. http://vejledninger.dsam.dk/akut/ ?mode=chart\&chartld=17 (accessed 9-7-2013).

22. Andersen TF, Madsen M, Jorgensen J, et al. The Danish National Hospital Register. A valuable source of data for modern health sciences. Dan Med Bull 1999;46:263-8.

23. Lynge E, Sandegaard JL, Rebolj M. The Danish National Patient Register. Scand J Public Health 2011;39:30-3.

24. Johannesdottir SA, Horváth-Puhó E, Schmidt M, et al. Existing data sources for clinical epidemiology: the Danish National Database of Reimbursed Prescriptions. Clin Epidemiol 2012;4:1-11.

25. Januel JM, Chen G, Ruffieux C, et al. Symptomatic in-hospital deep vein thrombosis and pulmonary embolism following hip and knee arthroplasty among patients receiving recommended prophylaxis: a systematic review. JAMA 2012;307:294-303.

26. Pedersen AB, Mehnert F, Johnsen SP, et al. Venous thromboembolism in patients having knee replacement and receiving thromboprophylaxis: a Danish population-based follow-up study. $J$ Bone Joint Surg Am 2011;93:1281-7.

27. Singh JA, Jensen MR, Harmsen WS, et al. Cardiac and thromboembolic complications and mortality in patients undergoing total hip and total knee arthroplasty. Ann Rheum Dis 2011;70:2082-8.

28. Bateman BT, Schumacher HC, Wang S, et al. Perioperative acute ischemic stroke in noncardiac and nonvascular surgery: incidence, risk factors, and outcomes. Anesthesiology 2009;110:231-8. 
29. Mortazavi SM, Kakli H, Bican O, et al. Perioperative stroke after total joint arthroplasty: prevalence, predictors, and outcome. J Bone Joint Surg Am 2010;92:2095-101.

30. Lalmohamed A, Vestergaard P, Klop C, et al. Timing of acute myocardial infarction in patients undergoing total hip or knee replacement: a nationwide cohort study. Arch Intern Med 2012;172:1229-35.

31. Sobieraj DM, Lee S, Coleman $\mathrm{Cl}$, et al. Prolonged versus standard-duration venous thromboprophylaxis in major orthopedic surgery: a systematic review. Ann Intern Med 2012;156:720-7.

32. Pedersen $A B$, Sorensen HT, Mehnert F, et al. Risk factors for venous thromboembolism in patients undergoing total hip replacement and receiving routine thromboprophylaxis. J Bone Join Surg Am 2010;92:2156-64.

33. Husted $\mathrm{H}$, Jensen $\mathrm{CM}$, Solgaard $\mathrm{S}$, et al. Reduced length of stay following hip and knee arthroplasty in Denmark 2000-2009: from research to implementation. Arch Orthop Trauma Surg 2012;132:101-4

34. Warwick D, Friedman RJ, Agnelli G, et al. Insufficient duration of venous thromboembolism prophylaxis after total hip or knee replacement when compared with the time course of thromboembolic events: findings from the Global Orthopaedic Registry. J Bone Joint Surg Br 2007;89:799-807.

35. Poultsides LA, Gonzalez DV, Memtsoudis SG, et al. Meta-analysis of cause of death following total joint replacement using different thromboprophylaxis regimens. J Bone Joint Surg Br 2012;94:113-21.

36. Pedersen AB, Baron JA, Overgaard S, et al. Short- and long-term mortality following primary total hip replacement for osteoarthritis: a Danish nationwide epidemiological study. J Bone Joint Surg Br 2011;93:172-7.

37. Malviya A, Martin K, Harper I, et al. Enhanced recovery program for hip and knee replacement reduces death rate. Acta Orthop 2011;82:577-81.

38. Kirksey M, Chiu YL, Ma Y, et al. Trends in in-hospital major morbidity and mortality after total joint arthroplasty: United States 1998-2008. Anesth Analg 2012;115:321-7.

39. Severinsen MT, Kristensen SR, Overvad K, et al. Venous thromboembolism discharge diagnoses in the Danish National Patient Registry should be used with caution. J Clin Epidemiol 2010;63:223-8.

40. Khatod M, Inacio MC, Bini SA, et al. Pulmonary embolism prophylaxis in more than 30,000 total knee arthroplasty patients: is there a best choice? J Arthroplasty 2012;27:167-72.

41. Berwick DM. The science of improvement. JAMA 2008; 299:1182-4

42. Merkow RP, Ko CY. Evidence-based medicine in surgery: the importance of both experimental and observational study designs. JAMA 2011;306:436-7. 


\section{Correction}

Jørgensen CC, Jacobsen MK, Soeballe K, et al. Thromboprophylaxis only during hospitalisation in fast-track hip and knee arthroplasty, a prospective cohort study. BMJ Open 2013;3: e003965. The authors of this paper have found some mistakes in table 2. While all results, including those in table 2, are correct regarding the 'early discharge' cohort, the percentages and confidence intervals for the 'unsuccessful' early discharge cohort and the total cohort are incorrect. The authors believe this is due to a systematical error and have corrected the table below accordingly.

Table 2. Venous thromboembolic events, all thromboembolic events and mortality

\begin{tabular}{|c|c|c|c|}
\hline Outcomes & All procedures (n:4924) & Early discharge (n:4659) & $\begin{array}{l}\text { “Unsuccessful” early } \\
\text { discharge (n:265) }\end{array}$ \\
\hline $\mathrm{PE}$ & $10(0.20 ;[0.11-0.37])$ & $5(0.11 ;[0.05-0.25])$ & 5 (1.89; [0.81-4.34]) \\
\hline Any DVT & 16 (0.32; [0.20-0.52]) & $14(0.30 ;[0.18-0.50])$ & $2(0.75 ;[0.21-2.70])$ \\
\hline Proximal DVT & 11 (0.22; [0.12-0.40]) & $9(0.19 ;[0.10-0.36])$ & $2(0.75 ;[0.21-2.70])$ \\
\hline Any VTE & $26(0.53 ;[0.36-0.77])$ & $19(0.41 ;[0.26-0.64])$ & $7(2.64 ;[1.28-5.35])$ \\
\hline Any VTE (THA/TKA) & $\begin{array}{l}16 / 10(0.65 ;[0.40-1.05]) / \\
(0.45 ;[0.24-0.83])\end{array}$ & $\begin{array}{l}15 / 4(0.61 ;[0.37-1.00]) /(0.18 \\
[0.07-0.46])\end{array}$ & $\begin{array}{l}1 / 6(0.72 ;[0.13-3.96]) /(4.76 ; \\
[2.20-10.0])\end{array}$ \\
\hline Myocardial infarction & $8(0.16 ;[0.08-0.32])$ & $7(0.15 ;[0.07-0.31])$ & $1(0.38 ;[0.07-2.11])$ \\
\hline Ischaemic stroke & 8 (0.16; [0.08-0.32]) & $6(0.13 ;[0.06-0.28])$ & $2(0.75 ;[0.21-2.70])$ \\
\hline $\begin{array}{l}\text { Transient cerebral } \\
\text { ischaemia }\end{array}$ & $7(0.14 ;[0.07-0.29])$ & $7(0.15 ;[0.07-0.31])$ & $0(0.00 ;[0.00-1.43])$ \\
\hline Arterial embolus & 1 (0.02; [0.01-0.11]) & 0 (0.00; [0.00-0.08]) & 1 (0.38; [0.07-2.11]) \\
\hline Any TEE & 50 (1.02; [0.78-1.34]) & $39(0.84 ;[0.62-1.15])$ & 11 (4.15; [2.33-7.28]) \\
\hline Any TEE (THA/TKA) & $\begin{array}{l}29 / 21(1.18 ;[0.82-1.69]) / \\
(0.95 ;[0.62-1.45])\end{array}$ & $\begin{array}{l}27 / 12(1.10 ;[0.76-1.60]) / \\
(0.54 ;[0.31-0.94])\end{array}$ & $\begin{array}{l}2 / 9(1.44 ;[0.40-5.10]) /(7.14 ; \\
[3.80-13.01])\end{array}$ \\
\hline All-cause mortality & 17 (0.35; [0.22-0.56]) & 13 (0.28; [0.16-0.49]) & $4(1.51 ;[0.59-3.82])$ \\
\hline Unrelated to surgery & $4(0.08 ;[0.03-0.21])$ & $3(0.06 ;[0.01-0.20])$ & 1 (0.38; [0.07-2.11]) \\
\hline $\begin{array}{l}\text { Surgically related } \\
\text { mortality }\end{array}$ & $7(0.14 ;[0.07-0.29])$ & $4(0.09 ;[0.04-0.23])$ & 3 (1.13; [0.38-3.27]) \\
\hline Death of unknown cause & $6(0.12 ;[0.05-0.26])$ & $6(0.13 ;[0.06-0.28])$ & 0 (0.00; [0.00-1.43]) \\
\hline Fatal PE & 1 (0.02; [0.00-0.11]) & 1 (0.02; [0.00-0.12]) & 0 (0.00; [0.00-1.43]) \\
\hline $\begin{array}{l}\text { Fatal PE/death of } \\
\text { unknown cause }\end{array}$ & $7(0.14 ;[0.07-0.29])$ & $7(0.15 ;[0.07-0.31])$ & $0(0.00 ;[0.00-1.43])$ \\
\hline $\begin{array}{l}\text { Any VTE or death of } \\
\text { unknown cause }\end{array}$ & 32 (0.65; [0.46-0.92]) & $25(0.54 ;[0.37-0.80])$ & 7 (2.64; [1.28-5.35]) \\
\hline $\begin{array}{l}\text { Any TEE or death of } \\
\text { unknown cause }\end{array}$ & 56 (1.14; [088-1.48]) & 45 (0.97; [0.73-1.29]) & 11 (4.15; [2.33-7.28]) \\
\hline
\end{tabular}

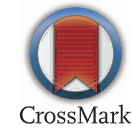

BMJ Open 2014;4:e0039365corr. doi:10.1136/bmjopen-2013-003965corr1 